\title{
A review of clinical profile, complications and antibiotic susceptibility pattern of extensively drug resistant (XDR) Salmonella Typhi isolates in children in Karachi.
}

\author{
Saba Shahid ( $\sim$ saba.shahid@tih.org.pk) \\ INDUS Hospital \\ Marvi Mahesar \\ INDUS Hospital \\ Nida Ghouri \\ INDUS Hospital \\ Saba Noreen \\ INDUS Hospital
}

Research article

Keywords: XDR Enteric fever, seasonality, children, antibiotics

Posted Date: August 18th, 2020

DOl: https://doi.org/10.21203/rs.3.rs-51063/v1

License: (c) (i) This work is licensed under a Creative Commons Attribution 4.0 International License.

Read Full License

Version of Record: A version of this preprint was published at BMC Infectious Diseases on September 3rd, 2021. See the published version at https://doi.org/10.1186/s12879-021-06599-2. 


\section{Abstract}

Background: Enteric fever is a systemic infection, which can be caused by Salmonella enterica; Typhi and Paratyphi A. Over a period of time Salmonella Typhi has developed resistance to many antibiotics which has resulted in emergence of extensively drug resistant (XDR) enteric fever. WHO estimated 5274 cases of XDR Enteric fever in Karachi from November 2016 to December 2019. This study aims to determine clinical course, complications and outcomes of XDR enteric fever among the pediatric population coming to Indus Hospital

Methods: A retrospective chart review of pediatric patients (aged 1 month-15 years) seen in Indus Hospital between July 2017 to December 2018 was conducted. A pre-designed data abstraction form was used to record detailed information about seasonality and distribution of cases, demographic details, signs and symptoms, clinical course, treatment, complications and final outcomes of the cases treated for XDR Enteric fever

Results: Six hundred and eighty children were included in the study. The median (IQR) age of the patients was $5(2-8)$ years. More than half $(n=391,57.5 \%)$ of the patients were males. Most common clinical manifestations included fever vomiting and diarrhea which were noted in 680 (100\%), 242 (35\%) and 174 patients (25\%) Final outcomes of 270 (39.7\%) patients have been recorded; 351 (51.6\%) were lost to follow up, 52 (7.6\%) were referred out and 7 (1\%) left without medical advice. 266 (39.1\%) patients were cured and 4 children (0.6\%) expired. Seventy eight patients (82\%) and 15 patients (16.3\%) got cured on Azithromycin and Meropenum alone while 157 patients got cured on combination of drugs.

Conclusion: Our review indicated that children under 5 years of age were affected more with XDR Enteric fever. Meropenum and Azithromycin, either alone or in combination were the most effective antibiotics for treating XDR Enteric fever in children coming to Indus hospital

\section{Background}

Enteric fever is a systemic infection, which can be caused by two serotypes of the gram-negative bacteria Salmonella enterica; Typhi and Paratyphi A (1). The disease is estimated to affect approximately 11 to 21 million individuals globally on an annual basis and has a high mortality rate (2). Recent data showed that globally 200,000 deaths result annually due to enteric fever (3). The burden of disease of enteric fever is the highest in Asia; $93 \%$ of the global cases are reported from within this region (3). Southeast Asia has the third highest incidence within the region, with approximately 110 cases / 100000 population. The estimated incidence of enteric fever in Pakistan was found to be 413 / 100000 population in children aged 2-4 years and 573 / 100000 population in children aged 5-15 years. (1)

Over a period of time, Salmonella Typhi has developed resistance to many antibiotics which has resulted in emergence of Multi-drug resistant (MDR) Salmonella Typhi (S. Typhi) strains. These strains have shown resistance to both first and second line antibiotics, namely Ampicillin, Chloramphenicol, Cotrimoxazole and fluoroquinolones (4). This strain of enteric fever has been endemic in countries like 
Pakistan, India, Nepal and Bangladesh since the 1980s (5). A review of antimicrobial resistance of S. Typhi and Paratyphi A conducted in Pakistan from year 2009 till 2011, showed increasing frequency of MDR S. Typhi ( $91.7 \%$ ) as well as two cases of S.Typhi which were resistant to cephalosporin resistance (4).

In November 2016, a massive outbreak of ceftriaxone-resistant S. Typhi was reported among children residing in Hyderabad, Pakistan. Around 486 cases were reported and consumption of contaminated drinking water was linked with the infection. These strains were called extensively drug resistant (XDR) S.Typhi as they showed resistance not only to first and second line antibiotics but also to third generation Cephalosporins. Drug sensitivity pattern of XDR Typhoid strain showed sensitivity to either Carbapenems or Azithromycin (6). Since the outbreak of XDR Enteric in Hyderabad, many other cases of XDR Enteric fever have been reported. WHO estimated 5274 cases of XDR Enteric fever in Karachi from November 2016 to December 2019 (7).

XDR Enteric fever is a new strain and may have unique clinical manifestations and outcomes compared to MDR Enteric infection. There is scarcity of data on clinical features and response to treatment in children suffering from XDR Enteric fever both nationally and in other parts of world. Therefore this study is done to determine clinical course, complications and outcomes of XDR enteric fever among the pediatric population coming to Indus Hospital. Primary end points include, clinical manifestations, complications, response to treatment and final outcome of the participants. Secondary end points included seasonality of the infection and geographic distribution of the cases.

\section{Methods}

\section{Study design and data collection}

A retrospective chart review was conducted of medical records of children who were treated for XDR Enteric fever at The Indus Hospital from 1st July' 2017 to 31st December' 2018. All children, with ages ranging from 1 month to 15 years, were included in the study. The data was extracted through the Health Management Informatics System (HMIS); data of all the children confirmed of having XDR Enteric fever, on basis of culture and sensitivity reports were included in the study.

A pre-designed data abstraction form was used to record detailed information about seasonality and distribution of cases, patient demographics, signs and symptoms, clinical course, treatment, complications and final outcomes. Laboratory parameters were also recorded to determine the severity and course of the disease; these included complete blood count (CBC), liver function test (LFTs), serum electrolytes, Prothrombin Time (PT), Activated Partial Thromboplastin Time (APTT), Urea and Creatinine. Data of complications like, cholecystitis, mesenteric lymphadenopathy, ascites and pleural effusion, was taken from ultrasound reports. Complications were defined as XDR infection with sign, symptoms or lab evidence of hepatitis, cholecystitis, encephalitis, mesenteric lymphadenitis, shock, ascites, pleural effusion and aphasia 
Microbiological culture of venous blood was performed using $5 \mathrm{ml}$ of blood, drawn under aseptic measures. BacT/Aert culture bottles were used to collect blood samples (8) Antibiotic susceptibility for a group of antibiotics was tested using Kirby-Bauerdisk disc diffusion method on Muller-Hinton agar with standard antimicrobial disks using Clinical Laboratory Standards Institute guidelines.

\section{Case definitions}

\section{Renal impairment}

Increased creatinine $>1.5$ times upper limit of normal or decreased in urine output $<0.5 \mathrm{ml} / \mathrm{kg} /$ hourr for 6 hours (9)

\section{Hepatitis}

Deranged Liver function tests with ALT more than twice the reference value with or without hyperbilirubinemia, impaired coagulation and hypoglycemia

\section{Cholecystitis}

Evidence of inflamed gall bladder sludge in gall bladder on ultrasound

\section{Hematological complications}

High or low level of hemoglobin, leucocyte or platelet count according to the given lab references.

\section{Encephalopathy}

Changes in mental status, confusion or stupor with normal CSF findings

\section{Encephalitis}

Changes in mental status, confusion or stupor or signs of meningeal irritation with abnormal CSF findings

\section{Diarrhea}

Presence of loose or watery stools 3 times or more per day (10) 


\section{Statistical analysis}

Data was analyzed using SPSS version 24. Descriptive statistics were run for all continuous variables, exploring Skewness and Kurtosis. Mean with standard deviation was reported for normal distribution, while median with interquartile range was reported for skewed distribution. For normal distribution, normality was confirmed by applying T-test. For skewed distribution, Mann-Whitney test was applied to determine significance. For categorical variables, frequencies were determined. Significance of correlations was determined by applying statistical tests; $p$ value $<0.05$ was taken as significant

\section{Results}

1518 patients had blood culture positive for enteric fever during the study period. Out of these 1341 patients were children and 177 patients were adults. Out of 1341 pediatric patients, 680 met our inclusion criteria and were included in the final analysis. More than half $(n=391,57.5 \%)$ of the patients were males. The median (IQR) age of the patients was $5(2-8)$ years, with minimum and maximum 0 day to 14 years. Out of these patients, majority, 612 (90\%), directly presented to ER, followed by OPD, 65(9.6\%). Around a hundred $(n=101,14.9 \%)$ patients got admitted in hospital, out of which 98 (97\%) patients were admitted in ward and the rest were admitted in ICU for treatment (Table 1).

Maximum number of children, 540 (79\%) came from district East (Fig. 1). 2 seasonal peaks were identified in year 2018. One was in February-May 2018 and second peak was observed in August-October 2018 (Fig. 2).

Most common clinical manifestations included fever vomiting and diarrhea which were noted in 680 (100\%), 242 (35\%) and 174 patients (25\%) (Table 1). Most frequent complications observed included hyponatremia $(n=74,11 \%)$, acidosis $(n=75,9 \%)$, hypokalemia $(n=59,9 \%)$ and bicytopenia $(n=67,10 \%)$ (Table 2). One case of vertical transmission was observed and the newborn died of XDR enteric infection. Four cases of encephalitis were identified, out of which 3 children had aphasia on presentation. All children with encephalitis had full recovery without any residual weakness or speech difficulty. One child developed multi organ dysfunction and pulmonary hemorrhage.

Final outcomes of 270 (39.7\%) patients were recorded; 351 (51.6\%) were lost to follow up, 52 (7.6\%) were referred out and $7(1 \%)$ left without medical advice. $266(39.1 \%)$ patients were cured and 4 children $(0.6 \%)$ expired (Table 1). Response to antibiotics was observed in 252 patients, 95 patients got cured on single drug while 157 patients got cured on combination of drugs. Seventy eight patients (82\%) and 15 patients (16\%) got cured on Azithromycin and Meropenum alone (Table 2). Azithromycin was the most common antibiotic used for the treatment of XDR, it was administered to $274(61.2 \%)$ patients, followed by Ceftriaxone and Meropenum, which were administered to 198 (44.2\%) and 137 (30.6\%) patients, respectively

Mortality was observed in 4 children, including a newborn. Out of those who died, two were on a single antibiotic, Meropenum, while the other two were on a combination of antibiotics (e.g. Meropenum and 
Ceftriaxone). Patients who died developed complications, such as, bradycardia, respiratory distress, hypoglycemia and sepsis, electrolyte imbalance, encephalitis, and pulmonary hemorrhage.

\section{Discussion}

Enteric fever caused by S. Typhi continues to pose as a health burden globally, with the incidence being highest in low to middle income countries (LMIC), due to poor infrastructure of public health (11). According to World Health Organization (12), Pakistan faced the largest outbreak of XDR enteric fever, in Hyderabad in November 2016 followed by a similar outbreak in Karachi. XDR Enteric is a novel strain of $\mathrm{S}$. Typhi which belongs to $\mathrm{H} 58$ lineage and has plasmid encoded resistance ans extended-spectrum $\beta$ lactamase (ESBL) gene which is responsible for resistance to both first and second line antibiotics (13).

An investigation of XDR enteric fever in Hyderabad (7) and in Islamabad (14) revealed that $56 \%$ and $33 \%$ affected children were under 5 years of age. This finding is consistent with our study, since we found that the median age of affected children was 5 years (IQR: 2-8 years). This higher incidence of XDR enteric fever amongst younger children could be explained by the fact that children have lower immunity and require lower bacterial dose for development of an infection (15). Most of the participants in our study belonged to East district of Karachi, probably because Indus Hospital lies in the catchment area of this district. Many residential areas located in district East of Karachi, comprise of peri-urban slums, having unhygienic conditions, poor sewerage facilities and consumption of pipe-borne portable water supply by the people. The high burden of disease in these areas could be due to contaminated drinking water and mixing of drinking and sewage water, a finding which was also observed in Hyderabad (6).

Enteric fever has been associated with considerable seasonal variations in different parts of world (16) In Pakistan MDR Enteric peaks have been noted in May-June and in October. The seasonality was linked to increased consumption of contaminated local drinks and ice-cream during hot season and post monsoon contamination of drinking water with rain water (17). We also observed 2 peak seasons of XDR Enteric cases, one in February-May 2018 and second in August-October 2018. This observation is contrary to previous epidemiological findings of Enteric fever in Karachi, which identified clear relation of Enteric fever with monsoon rains. Epidemiological survey has shown that Sindh province, including Karachi, remained generally dry throughout 2018 (18), which makes post monsoon contamination of water less likely in our study. However timing of peak cases in our study were very similar to those observed in Lahore in 2018. In Lahore increased numbers of cases of XDR Enteric fever were observed from January April 2018 (19), while XDR peak in our study was from February-May 2018. The most probable explanation for seasonal similarity of XDR cases between Karachi and Lahore, within same time period, could be because of intercity travelling of people.

In our study most successful, single drug was Azithromycin, which cured 78 cases (82\%) followed by Meropenem, which cured 15 cases (16\%). This finding is consistent with other studies, which also found better cure rates with Azithromycin and Meropenem $(20,21)$ Seventy six patients $(48 \%)$ were successfully treated wth combination of Azithromycin and Meropenum. We found synergistic effect of Azithromycin 
and Meropenum in achieving fever defervescence. Synergism among various antibiotics have been observed in MDR Enteric fever also (22). Eight children were cured on single drugs although they were resistant to those antibiotics. There is a possibility that these strains were sensitive to antibiotics but had higher MIC breakpoints and were interpreted as resistant strains in our labs, since we did not determine minimum inhibitory concentration (MIC) of the antibiotics used for treatment of XDR Enteric strains.

The strength of this study is that it is the only extensive study in our knowledge which examines the clinical course and outcomes of XDR strain of S. Typhi amongst the pediatric population within Pakistan. The study also determined response to antibiotics and complications in XDR Enteric fever. The limitations of this study includes lack of use of minimum inhibitory concentration (MIC) for culture and sensitivity, along with absence of molecular mapping, which would have provided a more comprehensive picture of the disease. We did not routiney perform stool cultures and hence could not identify carriers.

Retrospective data had missing information on socio demographics like drinking water quality, hygiene practices and number of people in household.

\section{Conclusion}

It can be concluded that children under 5 years of age were affected more with XDR Enteric fever. Meropenum and Azithromycin, either alone or in combination were the most effective antibiotics for treating XDR Enteric fever.We advocate increasing nation wide awarness about consumption of safe water, antibitic stewarship and immunization practices of children.

\section{Abbreviations}

XDR: Extensively drug resistant, MDR: multi drug resistance, WHO: World health organization, IQR: Inter quartile range, S. Typhi: Salmonella Typhi, HMIS: Health Management Informatics System, CBC: complete blood count, LFT: liver function test, PT: Prothrombin Time, APTT: Activated Partial Thromboplastin Time, ALT: alanine transaminase, CSF: cerebrospinal fluid

\section{Declarations}

\section{Ethics approval and consent to participate:}

Ethical approval was taken from the Institutional Review Board (IRB) of Interactive Research \& Development, registered with the U.S. Department of Health and Human Services, Office for Human Research Protections at The Indus Hospital. As per the IRB, written informed consent was not required from the patients involved in this study.

\section{Consent for publication:}

Not applicable 


\section{Availability of data and materials:}

The datasets used and/or analysed during the current study are available from the corresponding author on reasonable request.

\section{Competing interests:}

The authors declare that they have no competing interests.

\section{Funding:}

No funding was received for this research.

\section{Authors' contributions:}

SS and MM conceived idea of the study and participated in study design and write up. SN and ND carried out data collection, MM and ND assisted with statistical analysis. All authors were involved in the coordination of the study, drafting the manuscript and approving the final version.

\section{Acknowledgements:}

Not applicable

\section{References}

1. Mogasale V, Maskery B, Ochiai RL, Lee JS, Mogasale VV, Ramani E, et al. Burden of typhoid fever in low-income and middle-income countries: a systematic, literature-based update with risk-factor adjustment. Lancet Glob Health. 2014;2(10):e570-80.

2. Acosta CJ, Danovaro-Holliday MC, Baiqing D, Bhattacharya SK, Agtini MD, Bhutta ZA, et al. A study of typhoid fever in five Asian countries: disease burden and implications for controls. 2008;86(4):260.

3. Crump JA, Luby SP, Mintz ED. The global burden of typhoid fever. Bull World Health Organ. 2004;82(5):346-53.

4. Mandal S, Debmandal M, Pal NK. Antibiotic resistance of Salmonella enterica serovar Typhi in Kolkata, India, and in vitro experiments on effect of combined chemotherapy. Sci World J. 2012;2012:454059.

5. Akhtar S, Sarker MR, Jabeen K, Sattar A, Qamar A, Fasih N. Antimicrobial resistance in Salmonella enterica serovar typhi and paratyphi in South Asia-current status, issues and prospects. Crit Rev Microbiol. 2015;41(4):536-45. 
6. Qamar FN, Yousafzai MT, Khalid M, Kazi AM, Lohana H, Karim S, et al. Outbreak investigation of ceftriaxone-resistant Salmonella enterica serotype Typhi and its risk factors among the general population in Hyderabad, Pakistan: a matched case-control study. Lancet Infect Dis. 2018;18(12):1368-76.

7. Typhoid fever - Islamic Republic of Pakistan. World Health Organization; 201827 December 2018.

8. Wikler MA. Methods for dilution antimicrobial susceptibility tests for bacteria that grow aerobically: approved standard. J CLSI. 2006;26:M7-A.

9. Sutherland SM, Byrnes JJ, Kothari M, Longhurst CA, Dutta S, Garcia P, et al. AKI in hospitalized children: comparing the pRIFLE, AKIN, and KDIGO definitions. Clin J Am Soc Nephrol. 2015;10(4):554-61.

10. Organization WH. Diarrhoea [cited 2020 19th July 2020]. Available from: https://www.who.int/topics/diarrhoea/en/.

11. Radhakrishnan A, Als D, Mintz ED, Crump JA, Stanaway J, Breiman RF, et al. Introductory Article on Global Burden and Epidemiology of Typhoid Fever. Am J Trop Med Hyg. 2018;99(3_Suppl):4-9.

12. Organization WH, Journal WHOJEMH. Regional office for the Eastern Mediterranean. 2009.

13. Klemm EJ, Shakoor S, Page AJ, Qamar FN, Judge K, Saeed DK, et al. Emergence of an Extensively Drug-Resistant Salmonella enterica Serovar Typhi Clone Harboring a Promiscuous Plasmid Encoding Resistance to Fluoroquinolones and Third-Generation Cephalosporins. mBio. 2018;9(1):e00105-18.

14. Saeed N, Usman M, Khan EA. An Overview of Extensively Drug-resistant Salmonella Typhi from a Tertiary Care Hospital in Pakistan. Cureus. 2019;11(9):e5663.

15. Saha MR, Dutta P, Palit A, Dutta D, Bhattacharya MK, Mitra U, et al. A note on incidence of typhoid fever in diverse age groups in Kolkata, India. Jpn J Infect Dis. 2003;56(3):121-2.

16. Lin FY, Vo AH, Phan VB, Nguyen TT, Bryla D, Tran CT, et al. The epidemiology of typhoid fever in the Dong Thap Province, Mekong Delta region of Vietnam. Am J Trop Med Hyg. 2000;62(5):644-8.

17. Siddiqui FJ, Rabbani F, Hasan R, Nizami SQ, Bhutta ZA. Typhoid fever in children: some epidemiological considerations from Karachi, Pakistan. Int J Infect Dis. 2006;10(3):215-22.

18. Society PM. Monsoon 2018 Rainfall Update October 2018 [cited 2020 13th July 2020]. Technical Report No. 2/2018:[Available from: http://www.pmd.gov.pk/cdpc/monsoon2018rainfall.pdf.

19. Latif S, Zia A, Ali SB, Hafeez S. Extensively Drug Resistant Typhoid Fever Seen at Tertiary Care Hospital in Lahore. Inf Dis J of Pak.51.

20. Wong W, Al Rawahi H, Patel S, Yau Y, Eshaghi A, Zittermann S, et al. The First Canadian Pediatric Case of Extensively Drug-Resistant Salmonella typhi Originating from an Outbreak in Pakistan and its Implication for Empiric Antibiotic Choices. IDCases. 2019:e00492.

21. Chatham-Stephens K, Medalla F, Hughes M, Appiah GD, Aubert RD, Caidi H, et al. Emergence of extensively drug-resistant Salmonella Typhi infections among travelers to or from Pakistan-United States, 2016-2018. Morb Mortal Wkly Rep. 2019;68(1):11. 
22. Zmora N, Shrestha S, Neuberger A, Paran Y, Tamrakar R, Shrestha A, et al. Open label comparative trial of mono versus dual antibiotic therapy for Typhoid Fever in adults. PLoS Negl Trop Dis. 2018;12(4):e0006380.

\section{Tables}


Table 1

Demographic charaterstics, clinical feaures, investigations, complications and outcome of the patients

\begin{tabular}{|ll|}
\hline Demographic features & Number (\%) \\
\hline Male & $391(57.5)$ \\
\hline Female & $289(42.5)$ \\
\hline Age in years Medain (IQR) & $5(0-14)$ \\
\hline Place of presentation & $612(90)$ \\
\hline OPD & $65(9.6)$ \\
\hline Inpatient & $3(0.4)$ \\
\hline Clinical features & \\
\hline Fever & Number (\%) \\
\hline Vomitting & $680(100)$ \\
\hline Diarrhea & $242(35.6)$ \\
\hline Anorexia & $174(25.6)$ \\
\hline Cough & $140(20.6)$ \\
\hline Abdominal pain & $126(18.5)$ \\
\hline Bleeding per rectum & $125(18.4)$ \\
\hline Rash & $4(0.6)$ \\
\hline Urinary symptoms & $12(1.8)$ \\
\hline Hepatomegaly & $14(2.1)$ \\
\hline Splenomegaly & $63(9.3)$ \\
\hline Duration of illness Median (IQR) & $22(3.2)$ \\
\hline Lab investigations & $14(8-26.3)$ \\
\hline Severe Anemia (Hb $<5$ gm/dl) & Number (\%) \\
\hline Severe Thrombocytopenia $\left(<50,000 \times 10^{9} / \mathrm{L}\right.$ & $9(1)$ \\
\hline Raised CRP $>5$ & $24(3.5)$ \\
\hline Hyponatremia (severe + Moderate combined) & \\
\hline Hypokalemia (severe + Moderate combined) & \\
\hline
\end{tabular}




\begin{tabular}{|ll|}
\hline Demographic features & Number (\%) \\
\hline Acidosis & $75(9)$ \\
\hline Raised serum creatinine & $4(0.6)$ \\
\hline Complications & Number (\%) \\
\hline Bicytopenia & $67(10)$ \\
\hline Pancytopenia & $16(2.4)$ \\
\hline Hepatitis & $11(1.6)$ \\
\hline Cholcystits (on abdominal ultrasound) & $4(0.5)$ \\
\hline Mesentric lymphadenopathy (on abdominal ultrasound) & $11(2)$ \\
\hline Pleural effusion (on abdominal ultrasound) & $6(1)$ \\
\hline Peritoneal free fluid (on abdominal ultrasound) & $8(1)$ \\
\hline Shock & $5(0.7)$ \\
\hline Encephalopathy & $16(2.3)$ \\
\hline Encephalitis & $4(0.6)$ \\
\hline Aphasia & $4(0.6)$ \\
\hline Vertical & $1(0.1)$ \\
\hline Outcome & \\
\hline Cured & $266(39.1)$ \\
\hline Died & $4(0.6)$ \\
\hline Lost to follow up & $351(51.6)$ \\
\hline LAMA/referred out & $59(8.6)$ \\
\hline
\end{tabular}


Table 2

Response to antibitics

\begin{tabular}{|ll|}
\hline Cured with single drugs & $\mathbf{N}(\%)$ \\
\hline Ceftriaxone & $6(6.3)$ \\
\hline Cefixime & $6(6.3)$ \\
\hline Meropenum & $15(15.8)$ \\
\hline Azithromycin & $78(82)$ \\
\hline Ciprofloxacin & $1(1.1)$ \\
\hline Cured with multiple drugs & $\mathbf{N}(\%)$ \\
\hline Meropenum + Azithromycin & $79(50)$ \\
\hline Other drug combinations & $81(51.6)$ \\
\hline
\end{tabular}

Figures

\section{Karachi District}

\begin{tabular}{|l|r|}
\hline Karachi District & No of cases \\
\hline Karachi Central & 14 \\
\hline Karachi East & 540 \\
\hline Karachi South & 16 \\
\hline Karachi West & 22 \\
\hline Malir & 99 \\
\hline
\end{tabular}
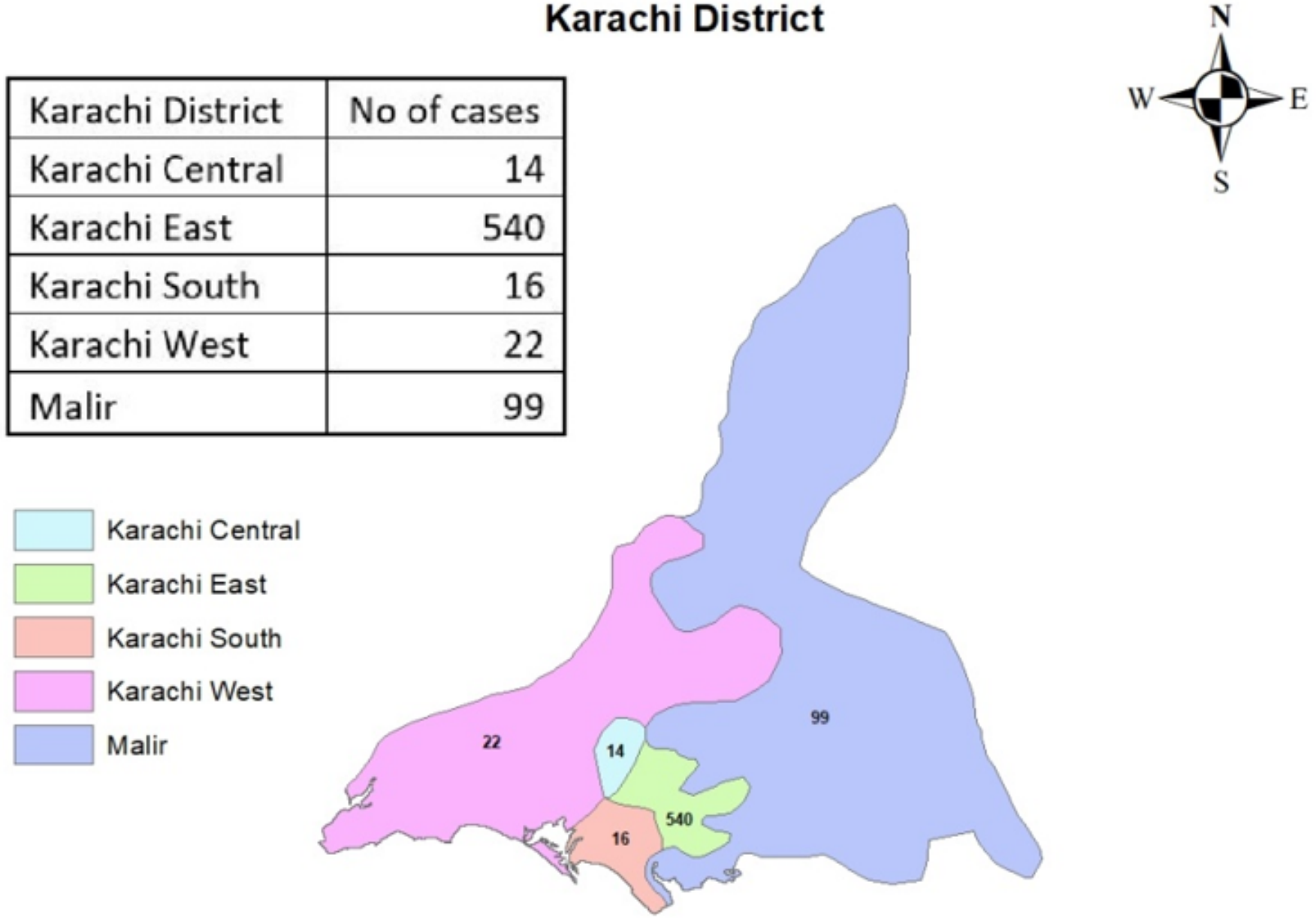
Figure 1

Geospatial map of XDR Enteric cases in Karachi

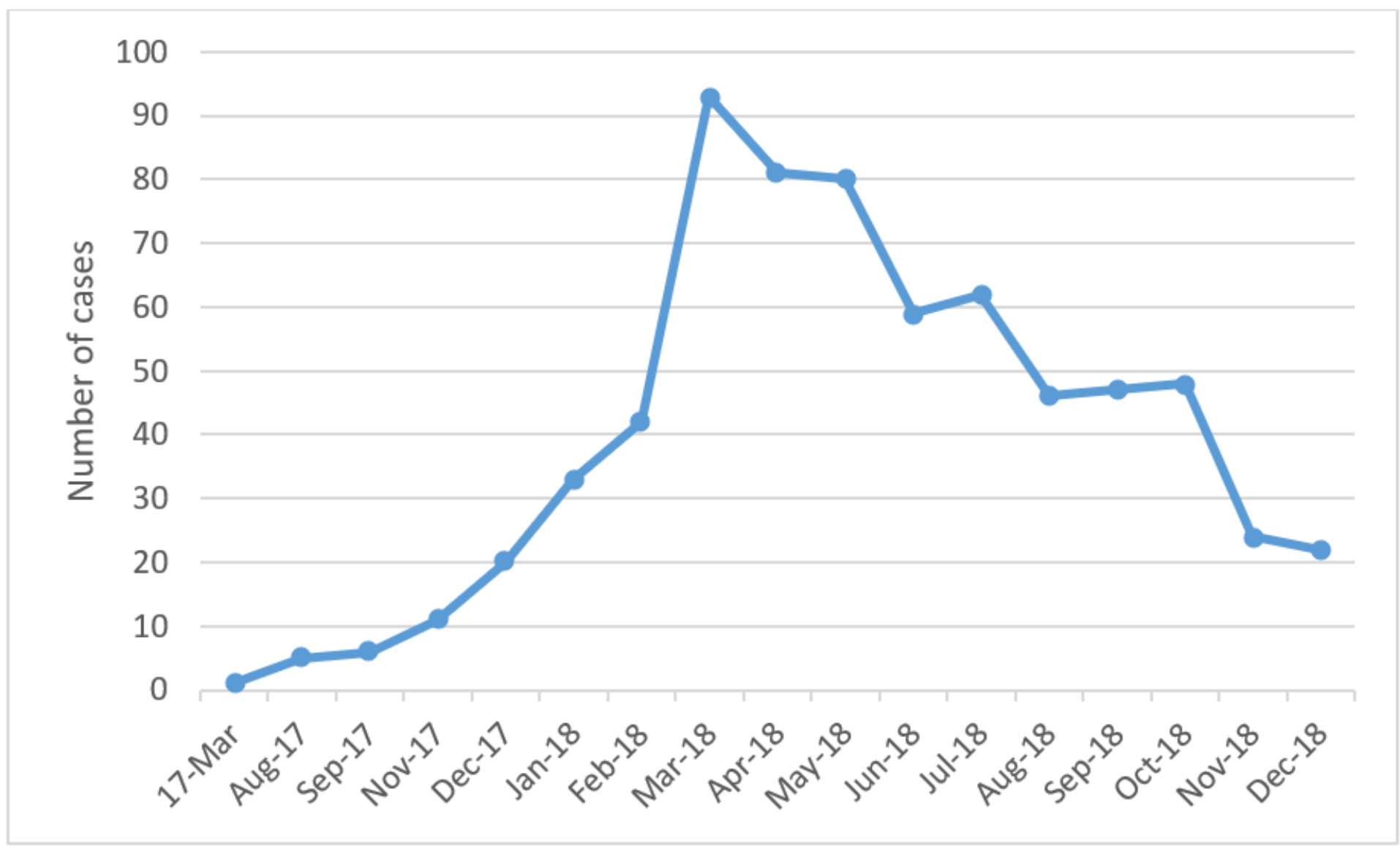

Figure 2

Seasonal pattern of XDR Enteric fever 